Ann. Biol. anim. Bioch. Biophys., 1977, 17 (5 A), 645-654.

\title{
Modifications quantitatives et qualitatives des lipides pilaires chez le rat en alimentation restreinte. Rôle joué par les hormones sexuelles.
}

\author{
J. ANDRÉ, Michelle CHASSAGNE
}

Station de Physiopathologie de la Nutrition, I. N. R. A.

Theix, Saint-Genès Champanelle 63110 Beaumont

Summary. Quantitative and qualitative changes of hair lipids in underfed rat. Role played by sexual steroids.

Adult rats are submitted to a 40 p. 100 alimentary restriction of ad libitum level over a 21-day period after initial hair delipidation. Hair lipids are determined by petroleum ether extraction on hair after total clipping. Fatty acid composition of hair lipids is studied by isothermal gas liquid chromatography.

The following results are obtained:

- in ad libitum fed animals, male rots have a higher hair lipid level than female rats $(p<0.001)$. Sexual differences in hair lipid fatty acids essentially concern anteiso-type acids and the C16/C18 ratio, higher in male $(p<0.005)$, and eisosenoic acid $(p<0.001)$ which is higher in female.

- undernutrition in male decreases seminal vesicle weight $(p<0.01)$ and hair lipid level $(p<0.01)$. Gonadectomy decreases sebaceous excretion by 55.3 p. 100 and abolishes feed restriction response. Testosterone supplementation, while restituting seminal vesicle weight to normal, also restores hair lipid level $(p<0.05)$.

- in female rats, undernutrition diminishes uterus weight $(p<0.05)$ and increases hair lipids, when drastic $(p<0.05)$. In ovariectomized rats, it tends to lower hair lipids (NS). Uterus weight restitution by physiological dose of estradiol is not accompanied by hair lipid level restitution (NS).

- qualitative changes in hair lipid fatty acids caused by undernutrition are not as extensive in female rats as in male rats. The more interesting qualitative parameter is the palmitate/stearate ratio which varies in male os does hair lipid level.

It is concluded that testosterone certainly plays a mediating role in the effect of undernutrition on sebaceous excretion in male, while estrogen effect in female is still to be investigated.

\section{Introduction.}

Les poils sont le lieu de dépôt de fractions imporfantes de composés sécrétés par les glandes sébacées et sudoripares. L'analyse pilaire chez le rat a ainsi permis de diagnostiquer des déséquilibres alimentaires antérieurs, minéraux (Brochart, 1975a, 
1975b) et protéiques (Brochart, 1975c). Afin d'étendre l'utilisation de l'analyse pilaire, dont un des avantages est de fournir une information rétrospective, à la détection de la sous-nutrition globale, nous avons envisagé le dosage des lipides déposés par excrétion sébacée. La justification d'un tel travail nous a été fournie par Pochi et al. (1970) qui ont montré que chez l'homme et la femme, le jeûne prolongé provoquait une chute de sécrétion de sébum. Dans des conditions expérimentales différentes, une sousalimentation prolongée chez la ratte s'est traduite par une augmentation de la quantité de lipides pilaires excrétés (Chassagne, non publié), lesquels sont d'origine sébacée (Ebling et Skinner, 1967) essentiellement. Ces résultats étaient en contradiction avec ceux de Pochi et al. (1970) mais une explication pouvait être donnée lorsque l'on sait que la sous-alimentation abaisse le taux d'hormones circulantes (Leathem, 1966) et que celles-ci ont une influence importante sur l'activité sébacée chez le rat (Ebling, 1974 ; Shuster et Thody, 1974), les androgènes la stimulant, les œstrogènes l'inhibant. Pochi ef al. (1970) avaient d'ailleurs eux-mêmes émis l'hypothèse de l'existence d'un médiateur hormonal androgénique dans l'action du jeûne sur la sécrétion sébacée.

Nous avons répété l'expérience effectuée précédemment chez la femelle en l'étendant au rat mâle et tenté de connaître le mécanisme d'action de la sous-alimentation sur l'activité sébacée en vérifiant s'il est effectivement de nature hormonale *.

\section{Matériel et méthodes.}

10 Plan expérimental et animaux (tabl. 1). Nous avons étudié l'influence de la sous-alimentation :

- sur des animaux entiers mâles et femelles : Expérience I ;

- sur des animaux castrés mâles et femelles : Expérience II ;

- sur des animaux entiers mâles et femelles supplémentés en hormones sexuelles : Expérience III.

Les rats utilisés étaient de souche Sherman, adultes, de même âge et sélectionnés de manière à ce que leurs poids initiaux soient les plus proches possible. Ils étaient continuellement en cage individuelle dans un environnement invariable afin d'éviter tout stress ou influence extérieure.

$2^{\circ}$ Régime alimentaire. Au cours d'une période préexpérimentale de 14 jours pour chaque expérience, les animaux mis en cages individuelles recevaient des quantités identiques d'aliment broyé ( $40 \mathrm{~g}$ pour les mâles, $30 \mathrm{~g}$ pour les femelles) afin de déterminer la consommation moyenne ad libitum. La mise en lots, et la restriction alimentaire étaient instituées à la fin de cette période pour une durée de 3 semaines.

3o Mesure quantitative des lipides pilaires.

Sepł jours après la mise en cage individuelle les poils étaient délipidés $\left(J_{0}\right)$ par bain des animaux dans un mélange éthanol-éther $1: 1 \mathrm{v} / \mathrm{v}$ pour uniformiser les quan-

* Ce travail a par ailleurs fait l'objet d'une thèse de $3^{2}$ cycle (André, 1976) où l'on trouvera des données originales ne figurant pas dans le présent article. 


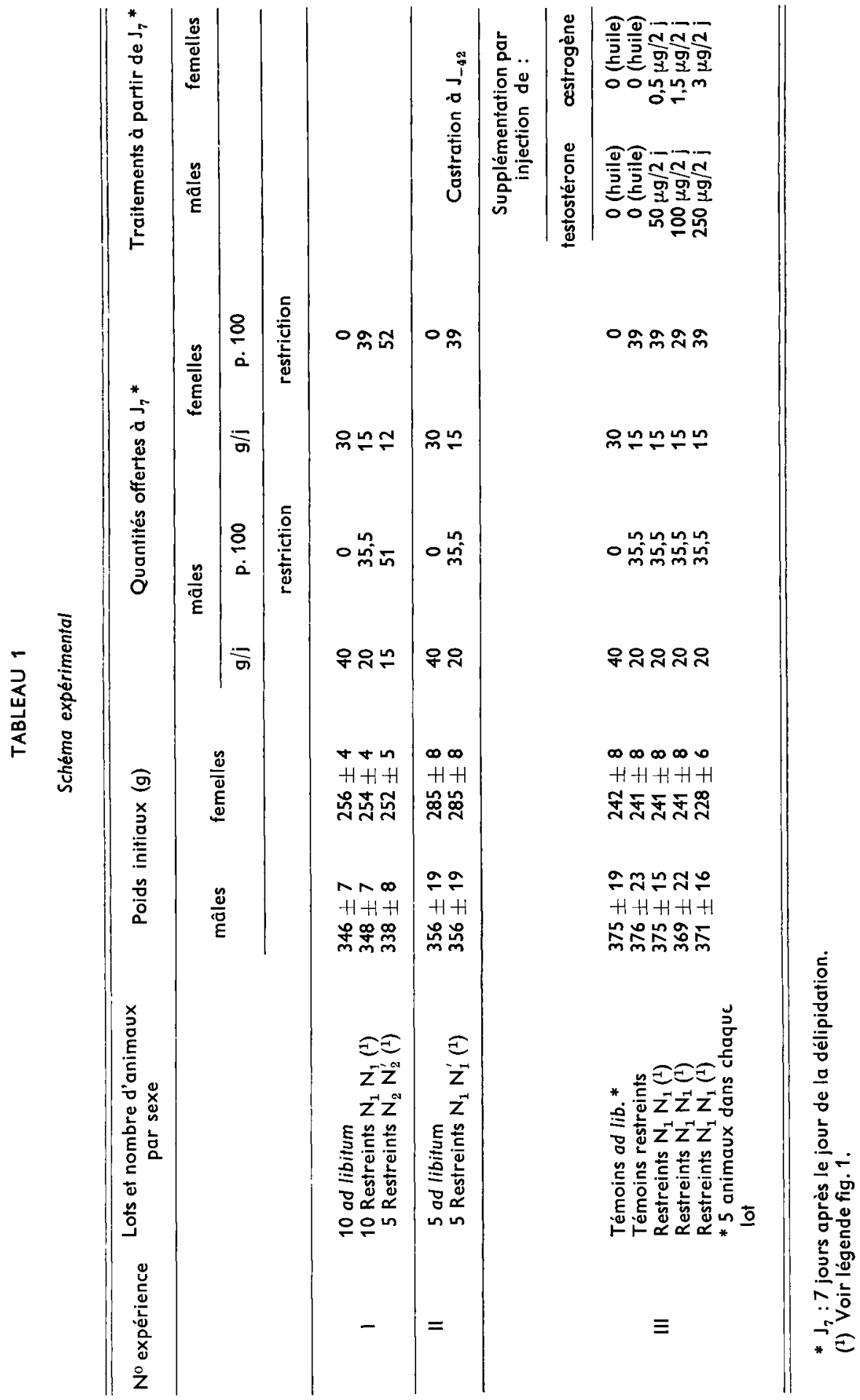


tités de lipides sébacés déposés sur les poils au moment de l'instauration des traitements (Archibald et Shuster, 1967, 1969, 1970). Une expérience préalable nous avait permis de constater que le plateau de relipidation est atteint 7 jours après lavage et que la variabilité individuelle est alors réduite. Les traitements étaient appliqués 7 jours après délipidation, donc à partir de $J_{7}$.

$A J_{28}$, les rats étaient sacrifiés et les poils tondus et conservés jusqu'au dosage.

Les lipides pilaires étaient dosés pondéralement après 3 extractions successives à l'éther de pétrole à raison de $50 \mathrm{ml}$ de solvant par gramme de poils. L'extrait organique était lavé par 0,2 fois son volume d'une solution de $\mathrm{NaCl} 0,85$ p. 100 pour éliminer les contaminants non lipidiques et récupérer les lipides acides.

Les lipides pilaires ont été exprimés en milligrammes de lipides extraits. Nous n'avons pas exprimé cette quantité de lipides par rapport à la masse pilaire ou à la masse corporelle car celles-ci varient du fait des traitements. Nous mesurons ainsi les lipides excrétés par un nombre constant de glandes sébacées.

$4^{0}$ Analyse des acides gras. La composition en acides gras longs $(\mathrm{nC}>12$ ) des lipides pilaires a été étudiée par chromatographie en phase gazeuse de leurs esters méthyliques obtenus par transméthylation pendant $3 \mathrm{~h}$ en présence du mélange méthanol-chloroforme-acide sulfurique $100: 100: 10 \mathrm{v} / \mathrm{v}$, sous reflux à $80^{\circ} \mathrm{C}$.

L'analyse était effectuée sur appareil Carlo Erba modèle Fractovap monocolonne, équipé d'un détecteur à ionisation de flamme. La colonne spiralée de $2 \mathrm{~m}$ de long et $2 \mathrm{~mm}$ de diamètre interne, était remplie de Chromosorb G Silanisé 80-100 mesh imprégné de 10 p. 100 de DEGS. Les conditions d'analyse étaient les suivantes : température du four $185^{\circ} \mathrm{C}$, température de l'injecteur et du détecteur $280^{\circ} \mathrm{C}$, débits d'azote, d'hydrogène et d'air de $30 \mathrm{ml} / \mathrm{mn}, 25$ et 300 respectivement.

Les acides gras avaient été identifiés au préalable par comparaison de leurs distances de rétention avec celles des esters méthyliques d'un mélange témoin. Les aires de pics étaient calculées par triangulation et le pourcentage de chaque acide du mélange obtenu en ramenant la surface du pic à la surface totale du chromatogramme.

$5^{\circ}$ Intervention sur le niveau hormonal.

Les animaux de l'expérience II étaient gonadectomisés 7 semaines avant le début des traitements $\left(J_{-42}\right)$.

Dans l'expérience III, le traitement hormonal débutait le $14 \mathrm{e}$ jour après la mise en cage individuelle. II consistait en l'injection intramusculaire tous les 2 jours de $0,05 \mathrm{ml}$ d'œnanthate de testostérone ou de benzoate d'œstradiol en solution dans l'huile d'olive neutre et stérilisée aux concentrations indiquées dans le tableau 1.

Nous avons estimé l'activité hormonale après traitement, par pesée des vésicules séminales pour les mâles et de l'utérus pour les femelles au moment du sacrifice.

Les différences et corrélations ont été considérées comme significatives pour les probabilités d'erreur $p<0,05\left(^{*}\right)$ et $p<0,01(* *)$. La signification des différences entre moyennes a été analysée par le test de Student-Fisher.

\section{Résultats.}

La consommation moyenne des animaux ad libitum a été de $31 \mathrm{~g} / \mathrm{j}$ pour les mâles et $24,5 \mathrm{~g} / \mathrm{j}$ pour les femelles. Les restrictions effectives ont donc été de 35,5 p. 100 et 
51 p. 100 pour les mâles et 39 p. 100 et 52 p. 100 pour les femelles au cours de la première expérience, le premier niveau de restriction seulement étant appliqué au cours des 2 suivantes.

Le jour du sacrifice, le poids des animaux restreints a toujours été significativement inférieur à celui des animaux ad libitum (fig. 1a, 2a, 3a).

10 Influence de la sous-alimentation.
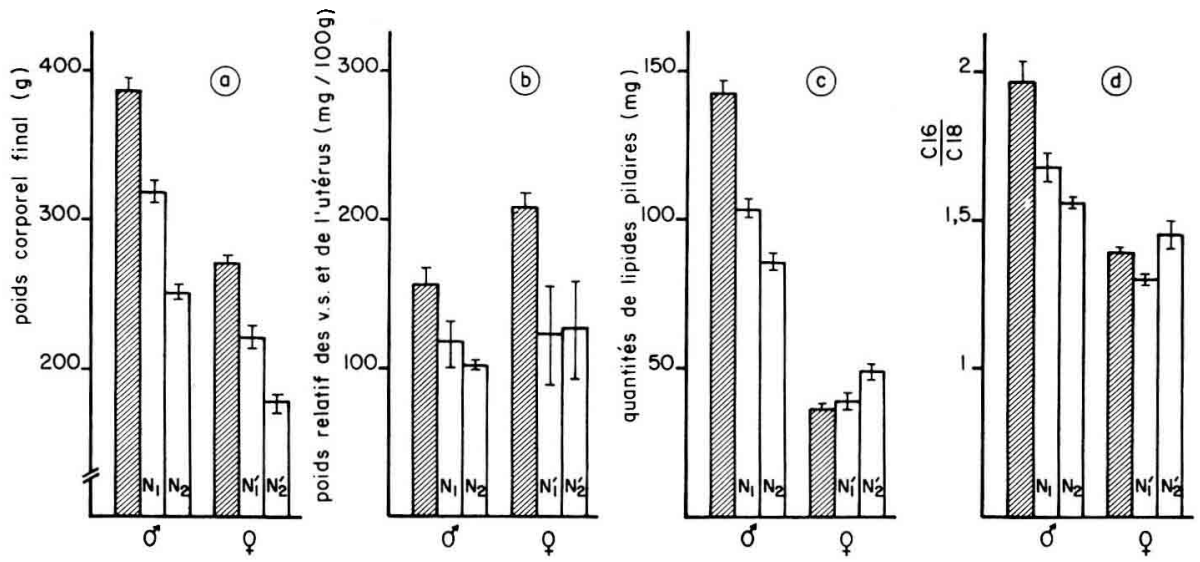

FIG. 1. - Influences du niveau olimentaire chez des rats mâles ef femelles entiers.

Q : Animaux ad libitum,

$\square$ : Animaux restreints,

$N_{1}: 1^{\mathrm{r}}$ niveau de restriction des rats mâles,

$N_{2}: 2^{e}$ niveau de restriction des rats mâles,

$\mathrm{N}_{1}^{\prime}$ : 1 r niveau de restriction des rats femelles,

$N_{2}^{\prime}: 2^{*}$ niveau de restriction des rats femelles.

Chez les animaux entiers mâles, le poids des vésicules séminales exprimé par $100 \mathrm{~g}$ de poids, est abaissé par la sous-alimentation, témoignage d'une réduction des androgènes circulants. Cette diminution est hautement significative pour le niveau de restriction le plus élevé (fig. 1b). La quantité de poils est peu modifiée.

Les lipides pilaires sont significativement réduits dès le premier niveau de restriction (fig. 1c) et leur composition en acides gras est modifiée.

Les pourcentages des acides palmitique (C16:0) et oléique (C18:1) sont abaissés, celui d'acide linoléique (C18:2) est augmenté significativement par la plus forte restriction. Le rapport palmitate/stéarate, proposé par Nikkari et Valavaara (1970) comme témoin de l'activité sébacée, varie dans le même sens que les lipides pilaires : il est abaissé par le plus fort niveau de restriction $(p<0,05)$ (fig. 1d), et il existe une corrélation positive $(r=+0,664, p<0,01)$ entre la quantité de lipides pilaires et ce rapport. Par contre, les variations de certains acides observées avec le premier niveau ne se retrouvent pas avec le second, ce qui semble paradoxal.

Chez les rats femelles, la sous-alimentation provoque une baisse du poids relatif de l'utérus (fig. 1b), laquelle n'est pas significative du fait sans doute des grandes 
variations individuelles. La quantité de poils n'est pas affectée. Les lipides pilaires extraits ne sont pas modifiés par le premier niveau de restriction mais sont augmentés $(p<0,05)$ par le second (fig. 1c).

Les variations de la composition en acides gras engendrées par la restriction sont beaucoup moins importantes chez les femelles que chez les mâles. En effet, la seule modification significative concerne l'acide pentadécanoïque (C15:0), au premier niveau de restriction seulement.

$2^{\circ}$ Influence de la castration.

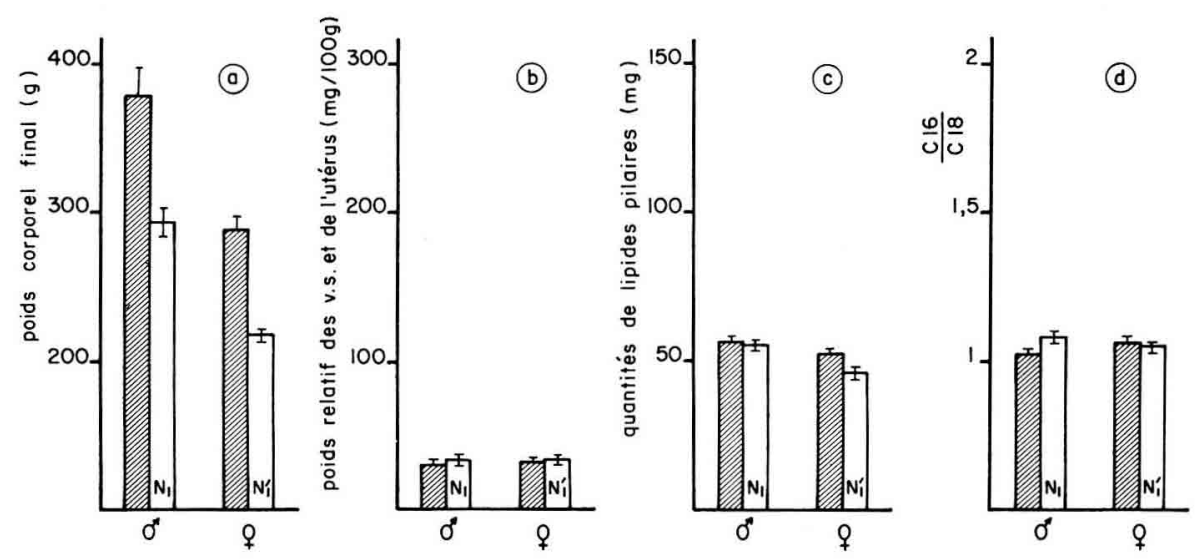

FIG. 2. - Influences du niveau alimentaire chez des rats mâles et femeiles castrés.

: Animaux ad libitum,

$\square$ : Animaux restreints,

$N_{1}$ : 1er niveau de restriction des rats mâles,

$N_{2}: 2^{e}$ niveau de restriction des rats mâles,

$N_{1}^{t}: 1$ er niveau de restriction des rats femelles,

$N_{2}^{\prime}: 2^{e}$ niveau de restriction des rats femelles.

La castration des rats mâles provoque une chute de poids relatif des vésicules séminales $(p<0,001)$ (fig. $2 b$ ) et des lipides pilaires $(p<0,001)$ (fig. $2 c$ ). Nous avons vérifié par ailleurs que l'opération en $\epsilon$ lle-même (pseudo-castration) ne modifiait pas le taux de lipides pilaires. Chez les animaux castrés, la composition en acides gras subit de profondes modifications : les acides 16-méthyl-heptadécanoïque (iso C18) et 18-méthyl-nonadécanoïque (iso $\mathrm{C20}$ ) sont augmentés (respectivement $p<0,001$ ef $P<0,005)$, les acides 12-méthyl-tridécanoïque (iso $C 14)(p<0,025)$, tétradécanoïque (C14:0) $(p<0,001)$, pentadécanoïque $(C 15: 0)(p<0,005)$, palmitique (C16:0) ( $P<0,001)$, 14-méthyl-hexadécanoïque (antéiso $C 17)(P<0,001)$, stéarique $(C 18: 0)(p<0,05)$ et oléique $(C 18: 1)(p<0,001)$ sont abaissés. Une quantité mesurable d'acide éicosanoïque ( $C 20: 0)$ apparaît en outre après castration. Le rapport C16:0/C18: 0 est significativement plus bas $(\mathrm{P}<0,001)$ chez les castrés que chez les entiers (fig. $2 d$ ).

La restriction alimentaire n'entraîne chez le mâle castré aucune modification de 
la quantité de lipides pilaires (fig. 2c) et seulement une faible modification de composition en acides gras par rapport aux rats normaux. Seuls le taux d'acide éicosanoïque $(\mathrm{C} 20: 0)$ et le rapport palmitate/stéarate sont significativement augmentés $(p<0,05)$ (fig. $2 d)$. Il semble donc que la restriction alimentaire conduit à une « castration physiologique », ce qui a motivé l'expérience III consistant à supplémenter en testostérone des mâles à niveau alimentaire restreint. La testostérone est sans doute l'intermédiaire par lequel la sous-alimentation agit sur l'activité sécrétoire des glandes sébacées. Il existe en effet une corrélation hautement significative ( $r=0,637 * * 15 \mathrm{~d}$.I.) entre le taux de lipides pilaires ef le poids des vésicules séminales des rats mâles entiers ad libitum et restreints de l'expérience 1.

$3^{\circ}$ Influence de la supplémentation hormonale chez les rats restreints.
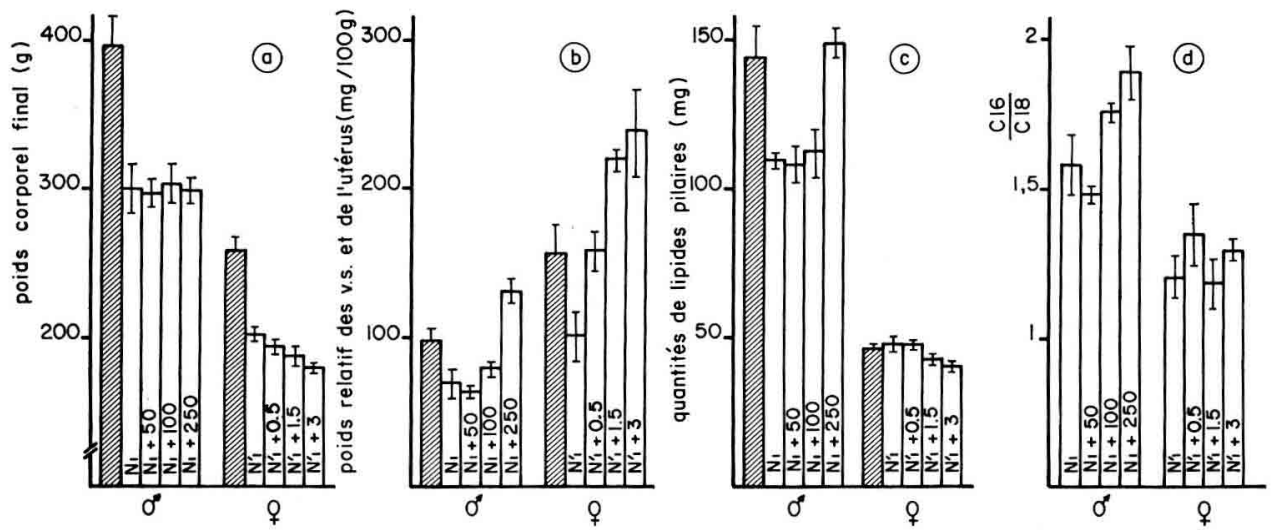

FIG. 3. - Influences de la supplémenfotion hormonale chez des rats entiers ef restreints.

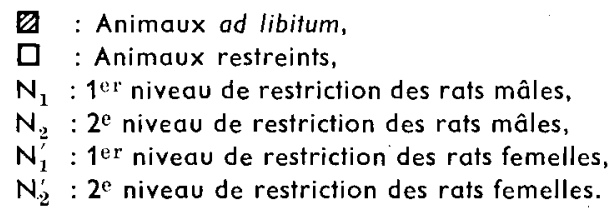

L'injection d'huile seule ne modifie pas l'effet dépressif de la restriction alimentaire sur le poids des vésicules séminales : le poids des vésicules séminales des rats restreints recevant de l'huile est significativement plus bas que celui des rats ad libitum recevant aussi de l'huile.

Injectée en quantités croissantes, la testostérone restaure progressivement le poids relatif des vésicules séminales chez les rats restreints. La plus forte concentration $(250 \mu \mathrm{g} / 2 \mathrm{j})$ permet même de dépasser le niveau des témoins ad libitum recevant de l'huile (fig. $3 b$ ).

La quantité de lipides pilaires chez les témoins restreints recevant de l'huile n'est pas significativement différente de celle des animaux ad libitum. Chez les animaux restreints supplémentés en testostérone, alors que les 2 premières doses modifient peu la quantité de lipides pilaires, l'injection de $250 \mu \mathrm{g} / 2 \mathrm{j}$ de testostérone élève les lipides 
pilaires à un niveau légèrement supérieur à celui des témoins ad libitum $(p<0,05)$ (fig. 3c).

L'injection de testostérone aux rats mâles restreints entraîne certaines modifications de la composition en acides gras : les pourcentages d'acides tétradécanoïque, pentadécanoïque ef palmitique $(\mathrm{C} 14: 0, \mathrm{C} 15: 0$ et $\mathrm{C} 16: 0)$ sont significativement augmentés, ainsi que le pourcentage de 12-méthyl-tétradécanoïque (antéiso $\mathrm{C15}$ ) et 14-méthyl-hexadécanoïque (antéiso C17) tandis que la proportion de 12-méthyl-nonadécanoïque (iso $\mathrm{C20}$ ) est diminuée pour les plus fortes doses. Le rapport palmitate/ stéarate, abaissé par l'injection de $50 \mu \mathrm{g}$, est augmenté par les deux autres doses, ces variations n'étant cependant pas significatives (fig. 3d).

Chez les femelles le poids relatif des utérus des rattes restreintes recevant de l'huile est inférieur à celui des rattes ad libitum, mais la différence observée n'est pas significative. Le poids relatif des utérus est restitué dès la première dose d'œstradiol injectée $(0,5 \mu \mathrm{g} / 2 \mathrm{j})$ et les concentrations les plus fortes l'amènent à un niveau anormalement élevé (fig. $3 b$ ).

Le niveau de restriction employé $(15 \mathrm{~g} / \mathrm{j})$ chez les témoins femelles, ne modifie pas l'excrétion des lipides. Celle-ci n'est pas altérée par l'injection de $0,5 \mu \mathrm{g}$ et $1,5 \mu \mathrm{g} /$ 2 j d'œstradiol. Par contre, on observe une diminution significative $(p<0,05)$ du taux de lipides pilaires chez les rattes restreintes recevant $3 \mu \mathrm{g} / 2 \mathrm{j}$ (fig. $3 c$ ).

Les modifications apportées par les œstrogènes dans les proportions d'acides gras sont peu importantes. Nous avons cependant constaté l'augmentation de l'acide 12-méthyl-tridécanoïque (iso $C 14)(p<0,05)$, de l'acide éicosanoïque $(C 20: 0)$ et de l'acide docosanoíque $(C 22: 0)(P<0,05)$ chez les rattes recevant $1,5 \mu \mathrm{g} / 2 \mathrm{j}$. L'injection de cette dose provoque aussi la baisse de l'acide 18-méthyl-nonadécanoïque (iso $\mathrm{C20}$ ) alors que $3 \mu \mathrm{g} / 2 \mathrm{j}$ entraîne la baisse des acides stéarique (C18:0) et 18-méthylnonadécanoïque (iso $\mathrm{C} 20$ ). Le rapport $\mathrm{C} 16 / \mathrm{C} 18$ ne montre pas de variation importante en réponse aux doses d'œstrogènes employées (fig. $3 d$ ).

\section{Discussion.}

Nos résultats confirment le dimorphisme sexuel observé par Nikkari (1965) chez les rats normaux au niveau des quantités de lipides pilaires et de leur composition en acides gras. Nous constatons que cette différence persiste dans la réponse de l'excrétion sébacée à la sous-alimentation : alors que les rats mâles normaux ont un taux de lipides pilaires et un rapport palmitate/stéarate très significativement supérieur aux femelles normales, la carence alimentaire se traduit chez le mâle par un abaissement des lipides pilaires et du rapport $\mathrm{C} 16 / \mathrm{C} 18$ et chez la femelle par une augmentation des lipides pilaires. La chute du poids relatif des vésicules séminales chez le mâle est hautement significative pour les animaux sous-alimentés, ce qui d'après Leathem (1966) correspond à une diminution du taux d'hormones sexuelles circulantes. Bien que non significative chez la femelle la chute du poids de l'utérus traduit le même phénomène. Lorsque l'on sait que la testostérone est un activateur de l'excrétion sébacée en augmentant à la fois les lipides excrétés et la synthèse de certains de leurs acides gras, alors que l'œstradiol en est un inhibiteur (Ebling et Skinner, 1967), il est logique que des effets identiques de la restriction sur le taux d'hormones sexuelles 
circulantes aient des conséquences opposées sur les lipides pilaires et certains de leurs acides gras si l'action des hormones sexuelles est déterminante dans l'activité sébacée.

C'est par un effet primaire sur les hormones sexuelles endogènes que la sousalimentation se manifeste sur l'activité des glandes sébacées. En effeł, après castration, et plus particulièrement chez le mâle, la sous-alimentation n'a plus d'effet sur le taux de lipides pilaires. Chez la femelle ovariectomisée, l'activité sébacée montre une fendance à la baisse. Enfin, si l’on rétablit les poids des vésicules séminales ou de l'utérus des rats restreints par injection d'hormones sexuelles, on restaure l'excrétion totale des lipides sébacés ef le rapport palmitate/stéarate au niveau de celui des mâles et des femelles normaux alimentés ad libifum.

La suppression de testostérone endogène d'origine testiculaire par castration entraîne une chute très significative du taux de lipides pilaires et du rapport C16/C18 tandis que l'injection de doses progressives de testostérone fait remonter la sécrétion des lipides au niveau de celle des animaux ad libitum recevant de l'huile, et le rapport C16/C18. II en est d'ailleurs de même d'autres acides gras sébacés qui suivent les mêmes variations. Les acides palmitique et oléique sont diminués significativement et de façon linéaire en fonction de la restriction appliquée. Ils sont aussi abaissés par castration.

A l'inverse, l'injection de testostérone aux rats mâles restreints permet de rétablir des proportions normales de palmitate et d'oléate, d'augmenter les pourcentages d'acides gras de la série antéiso, d'abaisser ceux de la série iso et de diminuer le pourcentage d'acide éicosénoïque (C20:1). Ces effets de la testostérone, observés lors d'injection de testostérone à des rats normaux par Nikkari (1965) témoignent de l'action de cette hormone, et plus particulièrement de son effet inhibiteur sur la synthèse d'acide éicosénoïque. Si l'on s'en tient à l'hypothèse de Nikkari et Valavaara (1970) selon laquelle la composition en acides gras des lipides pilaires dépend de l'activité sécréłoire des glandes sébacées, ces résultats sont logiques, puisque nous avons vu que la testostérone rétablit le taux de lipides pilaires des rats restreints.

Le rôle des cestrogènes dans la réponse à la sous-alimentation de l'activité sébacée des rats femelles est plus difficile à cerner au vu des expériences rapportées.

Pochi ef al. (1970) avaient obtenu chez la femme, comme chez l'homme, une chute de sécrétion de sébum lors du jeûne. Mais alors que ces auteurs mesuraient la sécrétion de sébum pendant $3 \mathrm{~h}$, nous mesurons la quantité de lipides excrétés pendant trois semaines ef déposés sur les poils. D'autre part, la restriction appliquée aux rattes ne crée pas de conditions identiques à celles d'un jeûne total. Enfin, l'augmentation de sécrétion sébacée provoquée chez la femelle par la restriction est insuffisante pour apprécier correctement l'effet de la supplémentation hormonale.

Le rétablissement du poids des utérus par la première dose d'œstradiol n'abaisse pas les lipides pilaires augmentés par la sous-alimentation. L'augmentation des lipides pilaires dans ces conditions ne semble donc pas induite en totalité par une chute d'œstrogènes. Les deux doses les plus fortes abaissent cependant nettement l'excrétion sébacée, en deçà même du niveau ad libitum. L'effet de ces doses bien que non physiologique dans la mesure où elles élèvent anormalement le poids des utérus trcduit le pouvoir inhibiteur des œstrogènes sur l'activité sébacée (Ebling et Skinner, 1967).

L'effet des œstrogènes sur la composition en acides gras des lipides pilaires semble très limité, mais il est intéressant de noter que les variations de certains acides, 
induites par l'œstradiol, vont dans un sens opposé à celles induites par la testostérone. Le manque de signification de ces variations ne nous permet pas de conclure.

Nous pensons que le niveau de restriction appliqué aux rattes, dont le seuil de réponse à la carence globale semble plus élevé que chez le mâle, est trop faible et n'a pas permis de mettre en évidence l'action des œestrogènes. Si l'augmentation observée ne peut être imputée à la diminution des œstrogènes circulants, il serait cependant difficile de trouver une explication à cette action.

Reçu en novembre 1976.

Accepté en janvier 1977.

\section{Références}

ARCHIBALD A., SHUSTER S., 1967. Bioassay of androgen using the rat sebaceous gland. J. Endocrin., 37, XXII.

ARCHIBALD A., SHUSTER S., 1969. The bioassay of androgens and antiandrogens using sebum secretion in the rat. Proc. roy. Soc. Med., 62, 887-888.

ARCHIBALD A., SHUSTER S., 1970. The measurement of sebum secretion in the rat. Brit. J. Derm., 82, 146-151.

BROCHART M., 1975a. Carence ef surcharge alimentaire en cuivre chez le rat : réponses plasmatique, hépatique, osseuse, pilaire. Ann. Rech. vét., 6, 231-235.

BROCHART M., 1975b. Latence ef amplitude des réponses plasmatique, osseuse, ef des fractions pilaires labile, stable et résiduelle à deux niveaux d'apport alimentaire en $\mathrm{K}, \mathrm{Na}, \mathrm{Ca}, \mathrm{Mg}, \mathrm{P}$ chez le rat. Ann. Rech. vét., 6, 337-344.

BROCHART M., 1975c. Influence de l'apport protéique alimentaire sur l'urée plasmatique ef l'urée pilaire labile chez le rat. Ann. Rech. vét., 6, 227-230.

EBLING F. J., 1974. Hormonal control and methods of measuring sebaceous gland activity. J. invest. Derm., 62, 161-171.

EBLING F. J., SKINNER J., 1967. The measurement of sebum production in rats treated with testosterone and œstradiol. Brit. J. Derm., 79, 386-393.

LEATHEM J. H., 1966. Nutritional effects on hormone production. J. anim. Sc., 25, 68-82.

NIKKARI T., 1965. Composition and secretion of the skin surface lipids of the rat; effects of dietary lipids and hormones. Scand. J. clin. Lab. Invest., 17 (suppl. 85), 1-40.

NIKKARI T., VALAVAARA M., 1970. The influence of age, sex, hypophysectomy and various hormones on the composition of the skin surface lipids of the rat. Hormones and sebum composition in the rat. Brit. J. Derm., 83, 459-472.

POCHI P. E., DOWNING D. T., STRAUSS J. S., 1970. Sebaceous gland response in man to prolonged total caloric deprivation. J. invest. Derm., 55, 303-309.

SHUSTER S., THODY A. J., 1974. The control and measurement of sebum secretion. J. invest. Derm., 62, 172-190. 